\title{
Influence of age, nutritional status and season on the reproductive system in wild male mink (Neovison vison)
}

\author{
Sara Persson • Britt-Marie Bäcklin • Hans Kindahl • \\ Björn Brunström • Ulf Magnusson
}

Received: 3 February 2010 /Revised: 7 February 2011 / Accepted: 16 February 2011 / Published online: 4 March 2011

(C) The Author(s) 2011. This article is published with open access at Springerlink.com

\begin{abstract}
The objectives of this study on the reproductive system of wild male mink (Neovison vison) were to determine the influence of age, nutritional status and season on reproductive traits and to produce baseline data that may be used in environmental monitoring programmes. The 117 male mink included in the study were killed by hunters all over Sweden (from latitude $56^{\circ} \mathrm{N}$ to $67^{\circ} \mathrm{N}$ ) during August to the end of April from 2005 to 2008. The weights of penis, testes, and epididymides were lower, the anogenital distance was shorter and the diameter of seminiferous tubules was less in juveniles compared with adult mink $(p<0.0001-p=0.0024)$. There was a positive effect $(p<0.05)$ of nutritional status on the baculum length and on the anogenital distance, but not on any of the other reproductive traits. The season of sampling influenced the penis, testes and epididymes weights and the diameter of seminiferous tubules $(p=0.008-p<0.0001)$. During spring, $86 \%$ of the
\end{abstract}

Communicated by C. Gortázar

S. Persson $\cdot$ H. Kindahl $\cdot$ U. Magnusson

Division of Reproduction,

Swedish University of Agricultural Sciences (SLU),

P.O. Box 7054, SE-750 07 Uppsala, Sweden

B.-M. Bäcklin

Department of Contaminant Research,

Swedish Museum of Natural History,

P.O. Box 50007, SE-104 05 Stockholm, Sweden

B. Brunström

Department of Environmental Toxicology, Uppsala University,

Norbyvägen $18 \mathrm{~A}$,

SE-752 36 Uppsala, Sweden

U. Magnusson $(\bowtie)$

SLU,

P.O. Box 7054, SE-750 07 Uppsala, Sweden

e-mail: ulf.magnusson@kv.slu.se mink had sperm in their epididymides, whereas the corresponding figure for autumn was $3 \%$; in addition, when the average diameter of the tubules reached $138 \mu \mathrm{m}$, sperm were present in the epididymides. Sperm morphology was analysed in 29 of the mink, and the percentage of morphologically defect sperm were generally low. Taken together, this study suggests that when assessing male reproductive data in wild mink populations, season must be taken into account as well as whether the individuals are juvenile or adult. In contrast, the nutritional status seems not to be of any major significance.

Keywords Mink $\cdot$ Reproduction $\cdot$ Season $\cdot$ Age $\cdot$ Nutrition

\section{Introduction}

Good reproductive performance of wild animal populations is crucial for their competitiveness. However, reproduction may be impaired by external factors such as infections and pollutants. These factors may cause a subtle, or sometimes even a dramatic, decline in population size. From a conservation biology perspective, it is important to recognize causes of population changes at an early stage. Animal reproductive performance may also serve as an indicator of environmental health. There are thus several good reasons to develop robust monitoring systems for the reproductive health in wild animals. As a contribution to establish such a monitoring system, the present paper points out factors that influence the reproductive system in wild male mink.

Well characterized sentinel species can be useful in monitoring of environmental contaminant exposure and effects (O'Brien et al. 1993; Beeby 2001). As a mammalian sentinel species in Sweden, the wild mink is a good candidate; it feeds on high trophic levels as it preys on fish, 
crayfish, amphibians and birds (Gerell 1967). Many pollutants end up in waters and the mink is presumably highly exposed to these pollutants due to its semi-aquatic way of life. The mink has a restricted home range (Gerell 1970; Dunstone 1993; Melero et al. 2008) and a widespread distribution in Sweden (Gerell 1970). Also, as the wild mink in Sweden is considered an invasive species, it may be hunted throughout the year in unlimited numbers. Another advantage is that mink carcasses are small enough to be easily handled and transported over long distances. The mink has previously been suggested as a suitable sentinel species in environmental health monitoring (Basu et al. 2007). In addition, the response of the mink to toxic substances is often similar to the responses of laboratory animals and humans (Calabrese et al. 1992), providing a large amount of comparative data.

Knowledge about the reproduction of farmed mink is extensive (Sundqvist et al. 1988, 1989), but less is known about the reproduction of wild mink. A direct comparison between reproduction in wild and farmed mink must be made with caution as the reproductive performance in domesticated animals is optimized and the reproductive seasonality reduced (Clark and Price 1981). A high reproductive performance in farmed mink is partly achieved through an optimised feeding regime with optimal composition of the feed (Bækgaard et al. 2008). In captivity, breeding for a desired production trait may also affect reproduction (Amador et al. 1996). Thus, one may not with certainty extrapolate data from domesticated to wild mink, regarding for example sexual maturation and photoperiodically controlled seasonality.

Hence, the primary objective of the current study was to investigate if factors known to influence the reproductive system in farmed mink or other mammalian species also affect the system in wild mink. A secondary objective was to generate baseline data for some reproductive traits that subsequently may be used in monitoring programmes for both environmental and reproductive health. Male wild mink were collected from all over Sweden (latitude $56^{\circ} \mathrm{N}$ to $67^{\circ} \mathrm{N}$ ). In particular, we wanted to unravel possible influence of age, nutritional status and season on the reproductive system.

\section{Material and methods}

\section{Sampling}

One hundred and seventeen mink were killed by local hunters during August to the end of April from 2005 to 2008. Mink were killed by a trap or shot while free-ranging and then frozen at approximately $-20^{\circ} \mathrm{C}$ as soon as possible after death. They were transported to the necropsy facilities at the National Veterinary Institute in Uppsala and thawed just before necropsy was performed. The age in years of each mink was determined by tooth cementum analysis on the mandibular fourth premolar (Matson's Laboratory, Milltown, Montana, USA). Age in months was then estimated from an assumed birth date in early May (Dunstone 1993). Three age categories were used in this study; juvenile (0-12 months old), 1-year old (1324 months) and more than 1-year old (more than 24 months). The number of mink in each group was 48 , 50 and 17, respectively. Hours of day-light at the specific capture date and site for each mink was used to construct three seasonal groups; autumn (17-9 h of day light before winter solstice), winter $(<9 \mathrm{~h})$ and spring $(9-17 \mathrm{~h}$ after winter solstice). The number of mink assigned to each group was 33, 45 and 37 , respectively.

\section{Post mortem procedure}

Before necropsy, the decomposition of the carcasses was assessed using a score system: (1) mild, for fresh specimens with no or few signs of decomposition; (2) moderate, when clear signs of autolysis is present; (3) substantial, for more advanced autolysis, organs start to lose firmness etc.; and (4) rotten, when organs are autolysed. The latter category was excluded from further analyses. The number of mink in each score category was 50, 59 and 6, respectively. Body weight was recorded and the body length was measured with a steel tape measure from nose to the base of the tail. Any gross lesion was recorded. Liver, spleen, adrenal and thyroid glands were weighed using a precision balance with a readability of $0.001 \mathrm{~g}$. The kidneys were dissected from their outer sheath and weighed. The subcutaneous fat between the hind legs on the ventral part of the abdomen was collected and weighed. Nutritional status was set as the weight of subcutaneous fat in relation to total body weight (gram subcutaneous fat $/ \mathrm{kg}$ body weight). Testes and epididymes weights were recorded separately. The baculum length and penis width and length were measured with a vernier caliper (accuracy $\pm 0.02 \mathrm{~mm}$ ). The penis was separated from the body where it enters the pelvis caudally and measured from the tip of the baculum to the cut off base. The preputium was removed before width measurement and weighing. The anogenital distance was measured with vernier calipers as the distance between anus and opening of the preputium. In a pilot study, triplicate measurements of the organs showed an intraassay variation less than $3.1 \%$.

One animal that was assessed as rotten and one that was severely damaged by a gunshot were excluded from the study. For eight mink with various types of abnormalities or lesions on the reproductive organs, no measurements on testes or epididymides were used in the Statistical analysis. Penis measurements were excluded for two mink; one with 
fractured baculum and the other with failure of preputial separation. All necropsies and measurements were made by the same person (Sara Persson).

Testis histology and sperm morphology

The right testis and epididymis were fixed in Bouin's solution for $24 \mathrm{~h}$, followed by storage in $70 \%$ alcohol until they were embedded in paraffin, sectioned and stained with hematoxylin and eosin. The sections were examined in a light microscope for presence of spermatozoa. The diameter of the seminiferous tubules was measured using a Leica DMR microscope (Leica Microsystems, Wetzlar, Germany) with a Deltapix Invenio camera and DpxView Pro software (DeltaPix, Maalov, Denmark). Five cross-sectioned tubules from each right testis were randomly selected and four diameter measurements were made on each tubule. The mean diameter of the tubules for each mink was used in the Statistical analysis, as well as the variation of tubular diameter within each testicle. Twenty-two mink that were poorly fixated or substantially decomposed were excluded from the histological evaluations on seminiferous tubular diameter.

A 25-gauge needle aspiration biopsy from the tail of the epididymis was taken and put in formol-saline (Hancock's solution). Sperm abnormalities were counted using phase contrast microscopy on 100-200 spermatozoa. For evaluation of sperm head morphology, William's-stained slides were made according to a staining method described by Lagerlöf (1934), and 150-500 spermatozoa were examined in a light microscope. Sperm morphology was evaluated blindly by the same technician. Twenty-nine mink were included in this study, as mink only produce sperm in connection to the breeding season and the aspiration procedure in some occasions failed to collect a sufficient number of spermatozoa.

\section{Statistical analysis}

Effects of age, sampling season and nutritional status on the reproductive traits, whole body measurements and internal organ weights were analyzed by least squares using the general linear model (GLM) procedure of SAS (SAS Institute Inc., Cary, NC, USA, version 9.02.01). Normality of data distribution was assessed using the univariate procedure of SAS. The model used for analyzing the effects was:

$\mathrm{Y}=\mu+\mathrm{AGE}+\mathrm{NUTRITION}+\mathrm{SEASON}+\mathrm{AGE}$

$$
\times \text { SEASON }+ \text { ERROR }
$$

Where: $Y$ is an observed value for the trait; $\mu$ is the population mean for the trait; AGE is a fixed effect due to age category of the mink; NUTRITION is an effect due to the nutritional status of the mink; SEASON is a fixed effect due to season of sampling; AGE $\times$ SEASON is a fixed effect due to the interaction between age category and season of sampling; ERROR is a random residual error term. $P$ values less than 0.05 were considered as significant. In a pre-analysis, the possible effect of latitude at sampling site on body size of adult animals according to Bergmann's rule (Bergmann 1847) was analysed in a univariate model. No effect was found and therefore latitude was excluded from the final model. Similarly, the possible effect of decomposition was subjected to preanalysis and was included in the final model for traits where there was such an effect. Unless indicated otherwise, data reported are least squares means \pm SD. In the attempt to generate solid baseline data for the traits, data were subjected to a power analysis using the GLMPOWER procedure of SAS. As there was no difference in least square mean between the two adult age categories for any reproductive trait but penile and baculum length, these two categories were fused before calculating baseline data and the power analysis (Table 2).

\section{Results}

Age analysis

The majority of the mink were juveniles or 1-year old $(85 \%$ together). The oldest mink was 5 years old. Juveniles were the most abundant age category collected during the early autumn months. During winter and spring, 1-year olds dominated. Older mink were caught occasionally throughout the hunting season.

Effects on reproductive organ characteristics by age, nutritional status and season of sampling

There was no effect of the degree of decomposition on the weight of the reproductive organs. The overall effects on the reproductive organs are shown in Table 1. Age had influence on all the reproductive traits $(p=0.03-p<0.0001)$. This was also true for season of sampling $(p=0.004-p<$ $0.0001)$, with the exception of anogenital distance. The weights of testes, epididymides and penis were lower, and the anogential distance was shorter in juveniles compared with the other age categories $(p<0.0001-p=0.0023)$. There was no difference for these traits between 1-year-old animals and those that were 2 years or older. Penile and baculum length increased $(p=0.0095$ and $p=0.0003$, respectively) from juveniles to the 1 -year-old mink, and increased further in animals that were 2 years old or more ( $p=0.012$ and $p=0.0182$, respectively). The testicular 
Table 1 Analysis of variance and data for reproductive traits, whole body measurements and some internal organ weights
$N . S$. not significant $(p<0.05)$, N.A. not applicable

${ }^{\mathrm{a}}$ Coefficient of determination for the statistical model (\%)

${ }^{b}$ The effect of age, nutritional status and season, respectively

${ }^{\mathrm{c}}$ The interaction between the effects of age and season

\begin{tabular}{|c|c|c|c|c|c|}
\hline \multirow[t]{2}{*}{ Dependent variable } & \multirow[t]{2}{*}{$R^{2 \mathrm{a}}$} & \multicolumn{4}{|c|}{ Source of variance (level of significance) } \\
\hline & & $\mathrm{Age}^{\mathrm{b}}$ & Nutritional status $^{\mathrm{b}}$ & Season $^{\mathrm{b}}$ & $(\text { Age } \times \text { season })^{\mathrm{c}}$ \\
\hline Baculum length & 53 & $<0.0001$ & 0.05 & 0.002 & N.S. ${ }^{d}$ \\
\hline Penis length & 43 & $<0.0001$ & N.S. & 0.008 & N.S. \\
\hline Penis weight & 52 & $<0.0001$ & N.S. & 0.001 & N.S. \\
\hline Testis weight & 66 & $<0.0001$ & N.S. & $<0.0001$ & 0.0005 \\
\hline Epididymis weight & 63 & 0.0002 & N.S. & $<0.0001$ & 0.009 \\
\hline Tubuli diameter & 71 & $<0.0001$ & N.S. & $<0.0001$ & 0.02 \\
\hline Tubuli diameter variation & 41 & 0.03 & N.S. & 0.004 & N.S. \\
\hline Anogenital distance & 29 & $<0.0001$ & 0.03 & N.S. & N.S. \\
\hline Body weight & 30 & N.S. & $<0.0001$ & 0.04 & N.S. \\
\hline Body length & 18 & N.S. & 0.005 & 0.04 & N.S. \\
\hline Subcutaneous fat & 88 & N.S. & N.A. & N.S. & N.S. \\
\hline Spleen weight & 20 & N.S. & 0.006 & N.S. & N.S. \\
\hline Liver weight & 16 & N.S. & N.S. & 0.003 & N.S. \\
\hline Kidney weight & 23 & N.S. & N.S. & 0.003 & N.S. \\
\hline Adrenal weight & 21 & N.S. & N.S. & 0.0009 & 0.01 \\
\hline Thyroid weight & 8 & N.S. & N.S. & N.S. & N.S. \\
\hline
\end{tabular}

tubular diameter and the variation in tubular diameter within the same testicle increased by age $(p<0.0001$ and $p=0.03$, respectively). There were not enough measurements made on tubular diameter in 2- to 5-year-old animals to make any comparison between the two adult age groups. The diameter of the seminiferous tubules correlated with testis weight $(R=0.948, p<0.0001)$. There was a positive effect $(p=0.05)$ by the nutritional status on the baculum length and the anogenital distance, but not on any of the other reproductive traits. Testicular, epididymal and penile weights increased from autumn to winter and also from winter to spring, but the length of penis and baculum did not increase significantly $(p>0.05)$ between winter and spring. The testicular tubular diameter and the variation in tubular diameter within the same testicle varied $(p<0.0001$ and $p=0.004$, respectively) over the seasons being largest in the spring. The baseline data for the reproductive traits with a sample power $>80 \%$ are shown by age category and season, respectively, in Table 2.

Effects on body weight and length and internal organ weights by age, nutritional status and season of sampling

Decomposition was associated with a reduced weight of liver $(p<0.0001)$, kidneys $(p=0.01)$ and thyroid gland $(p=$ $0.02)$. The overall effects on the whole body measurements and internal organ weights are shown in Table 1. Age showed no effect on these weights. The records of mink sampled in autumn were lower than those of mink from other seasons regarding the whole body measurements $(p=$ $0.05)$ and the weight of the spleen $(p=0.05)$, liver $(p=$ $0.0033)$, kidneys $(p=0.0124)$ and adrenals $(p=0.003)$. The nutritional status varied between 0 and $36 \mathrm{~g}$ subcutaneous fat $/ \mathrm{kg}$ body weight, with a mean of $11 \mathrm{gs}$.c. fat $/ \mathrm{kg}$ b.w. The
Table 2 Baseline data for traits with a sample power $>80 \%$ (least square mean and standard deviation)

\footnotetext{
${ }^{\text {a }}$ Paired organ weight
}

\begin{tabular}{|c|c|c|c|c|c|}
\hline \multirow[t]{2}{*}{ Variable } & \multicolumn{2}{|l|}{ Age } & \multicolumn{3}{|l|}{ Season } \\
\hline & Juvenile & Adult & Autumn & Winter & Spring \\
\hline Testes weight $^{\mathrm{a}}(\mathrm{g})$ & $1.1 \pm 1.3$ & $2.1 \pm 1.4$ & $0.7 \pm 0.6$ & $1.8 \pm 1.3$ & $2.9 \pm 1.3$ \\
\hline Epididymides weight $^{\mathrm{a}}(\mathrm{g})$ & $0.3 \pm 0.3$ & $0.5 \pm 0.4$ & $0.2 \pm 0.2$ & $0.4 \pm 0.3$ & $0.8 \pm 0.3$ \\
\hline Kidneys weight $^{\mathrm{a}}(\mathrm{g})$ & $7.8 \pm 0.8$ & $8.3 \pm 0.8$ & $7.6 \pm 1.8$ & $9.0 \pm 1.6$ & $9.9 \pm 2.4$ \\
\hline Penis weight $(\mathrm{g})$ & $1.2 \pm 0.6$ & $1.7 \pm 0.4$ & & & \\
\hline Anogenital distance $(\mathrm{mm})$ & $62.3 \pm 6.6$ & $68.2 \pm 6.1$ & & & \\
\hline Tubular diameter $(\mu \mathrm{m})$ & $91.6 \pm 43.4$ & $125.9 \pm 36.5$ & & & \\
\hline Penis length $(\mathrm{mm})$ & $54.8 \pm 5.2$ & $58.8 \pm 4.3$ & & & \\
\hline Baculum length (mm) & $41.1 \pm 4.1$ & $44.8 \pm 2.8$ & & & \\
\hline
\end{tabular}


nutritional status had a positive effect $(p<0.001)$ on the body weight and length and the weight of the spleen. The effects of age and season on nutritional status were calculated separately and no effect $(p>0.05)$ was observed. Season of sampling had influence on most traits and nutritional status on some $(p=0.04-p<0.0001)$. The model could only explain $8 \%$ of the variation in thyroid weight and none of the independent traits had any significant effect.

\section{Sperm evaluation}

During autumn, $3 \%$ of the mink had sperm present in their epididymidis. During winter and spring the percentage was 24 and 86, respectively. None of the juvenile mink had sperm during winter, but $34 \%$ of the adult mink (1 year old or more) did. During spring, $64 \%$ of the juveniles and $96 \%$ of the adults had sperm. It was found that when the average diameter of the tubules reached $138 \mu \mathrm{m}$, sperm were present in the epididymidis. The result of the sperm morphology analysis is given in Table 3 . There was no difference between mink caught during winter $(n=8)$ and mink caught during spring $(n=21)$ in frequency of any of the registered defects.

\section{Discussion}

This is, to our knowledge, the first field study on the reproductive system of wild male mink where influence of age, nutritional status and season is analyzed. Notably, the constructed multivariate analysis model explained the variation of the reproductive traits to a large extent. As mentioned in the Introduction, a direct comparison between farmed and wild mink might not be accurate. Reproduction in mink is influenced by photoperiod, but additional environmental factors seem to be of importance (Martinet et al. 1985; Tauson 1985, 1994). An example of this is the

Table 3 Sperm morphology, defect sperm (percent) $n=29$

\begin{tabular}{lll}
\hline & Mean \pm SD & Range \\
\hline Pathological heads $^{\mathrm{a}}$ & $5.5 \pm 4.1$ & $0.0-18.0$ \\
Mid-piece defects & $2.1 \pm 4.1$ & $0-20.0$ \\
Tail defects $^{\mathrm{b}}$ & $0.9 \pm 1.4$ & $0-4.8$ \\
Detached sperm heads $_{\text {Proximal cytoplasmatic droplets }}$ & $8.4 \pm 19.0$ & $0-99.0$ \\
Acrosome defects $^{\mathrm{c}}$ & $2.2 \pm 3.0$ & $0.0-13.0$ \\
\hline
\end{tabular}

${ }^{a}$ Pear-shaped, narrow-based, abnormal contour, lack of development, abnormal loose head, narrowness, variable size and abaxial implantation

${ }^{\mathrm{b}}$ Simple-coiled tail, tail coiled under the head and double-coiled tail

${ }^{\mathrm{c}}$ Knobbed, dented and protruded acrosome
Alaskan mink that has been shown to have a delayed breeding season in order to time it with the spawning of salmon (Ben-David 1997). At large, our data followed the general patterns of reproduction in farmed mink, but interestingly nutritional status did not seem to influence the reproductive measurements in wild male mink.

Notably, decomposition to the degree when organs start to lose firmness did not affect the weight of the reproductive organs. In contrast, the degree of decomposition has to be taken into account when analysing data about the weight of liver, kidney and thyroid glands.

In the attempt to generate reliable baseline data for the traits recorded per age category or season, not all traits reached a sample power above $80 \%$. Comparison of our baseline data with data from farmed mink is hampered because references are sparse and often based on relatively small sample sizes. However, paired testes weight in late March were reported to be on average $5.11 \mathrm{~g}$ (Onstad 1967), which is strikingly heavier than the paired weight in spring in this study ( $2.9 \mathrm{~g}$, Table 2$)$.

The dispersal of mink from different age categories varied throughout the sampling period. The number of collected juveniles was greatest during the autumn months. This is probably due to the dispersion that occurs when the mink family breaks up in late summer and the juveniles have to find their own home range (Gerell 1970). Also, the age distribution in a mink population may change in time as the mink reproduce seasonally.

When analyzing the effect of age, most of the reproductive traits, such as weight of testicles, epididymides and penis and the anogenital distance, seemed to differ between juveniles and adults, but not between 1-year olds and older mink. The high correlation between the diameter of the seminiferous tubules and the weight of the testicles suggests that the diameter of the tubules also follow the same age pattern. This assumption is supported by results on farmed mink from Onstad, who found no difference in tubular diameter in March between mink that were 1 and 2 years old (Onstad 1967). These and our data indicate that the development of most parts of the reproductive system in the wild male mink is completed after 1 year. However, the penile and baculum length seem to continue to increase with age. This has previously been found in other studies on baculum morphology on both wild and farmed mink (Elder 1951; Lechleitner 1954).

Nutritional status did not vary with season or age. Notably, there seems to be a large variation in the amount of subcutaneous fat of mink at all seasons. It can be speculated that the body-fat dynamics of the wild mink resembles that of the American marten (Martes americana), another lean-bodied mustelid with similar opportunistic lifestyle as the mink (Dunstone 1993; Ben-David et al. 1997). In a study by Buskirk and Harlow (1989), it was 
found that the American marten has sparse fat reserves that do not change with season and that omental mass was a good indicator of nutritional status. In a pilot study by us on wild mink, the subcutaneous fat and omental fat showed a strong positive correlation $(r=0.97)$ and the omental fat weighed on average $2 \mathrm{~g}$. This differs from farmed mink, where the omentum can weigh as much as on average $47 \mathrm{~g}$ (Mustonen et al. 2005). Interestingly, our data indicate that nutritional status of the animal is not a source of variance for the reproductive measurements in wild male mink. This can be interpreted as the fat reserves in the minks in the current study were not depleted to the extent that would cause changes in the measured reproductive traits.

The total body weight varied throughout the year; the mink weighed less during autumn but there was no difference in total body weight between winter and spring. This is in agreement with other studies in both wild and farmed mink that have found that the body weight is highest just before the mating season in March (Korhonen et al. 1989; Martinet et al. 1992; Dunstone 1993). In summary, our data indicate that body weight changes with season but fat depots do not. This might be explained by the anabolic effect of testosterone; as it gradually increases during the months prior to the breeding season (Pilbeam et al. 1979; Blottner et al. 2006), it alters the muscle mass and thus influences the total variation in body weight.

The reproductive organs of mink in captivity are known to be strongly influenced by seasonal variations (Enders 1952; Pilbeam et al. 1979), and this is thought to be essentially caused by changes in photoperiod (BoissinAgasse et al. 1986; Martinet et al. 1992). The decreasing day length during autumn stimulates testicular activity. After the breeding season in March, the testes regress and no activity is seen until next autumn. This means that latitude could be a potential bias factor in this study, so we decided to use photoperiod length at the specific date of capture to refine seasonality as a source of variance. Previous studies on reproductive seasonality in mink have mainly focused on the testes and sometimes on the epididymides. In this study, we found that the penis size also seems to be under the influence of season. Some internal organs such as the adrenal glands, liver and kidneys also varied by season; they weighed less during the autumn than during winter and spring. No macroscopical lesions were found that can explain these variations. A seasonal variation related to the breeding cycle in the histological structure of adrenal glands has previously been reported in male mink, but no consistent change was found in the total size of the gland (Ramos and Basrur 1972). The reason for seasonal variation in liver weight is unclear, but might be due to that the short days during autumn causes altered lipid and carbohydrate metabolism as suggested by Nieminen and co-workers (2001). In other species, seasonal variations in kidney weight have been described in, for example, sheep (Vuren and Coblentz 1985) and caribou (Dauphine 1975), but the causes were not identified. Possibly, the seasonal variation seen in internal organ weights is an adaption to the cold winter climate which coincides with the breeding season.

To our knowledge, there are no reports on sperm morphology in wild mink. A few studies have been performed on farmed mink, and in the most detailed study on mink with normal clinical appearance, $1.16 \%$ primary abnormalities originating from the testis and $22.77 \%$ abnormalities originating from the epididymis were found (Onstad 1967). Coiled tail is one of the most commonly reported defects (Onstad 1967; Aulerich et al. 1972), but in this study, the percentage coiled tails was very low. Here, we instead found a high percentage of abnormal acrosomes. However, this was not considered as a sperm defect, rather an artefact as the mink carcasses were frozen at approximately $-20^{\circ} \mathrm{C}$ and the spermatozoa were thus exposed to temperatures causing the membrane disruption. The frequency of all sperm defects was generally low. Comparing the data with domestic carnivores, the majority of the mink would probably have been fertile, although three mink had sperm defects that might impair fertility to some extent (Axnér and Linde Forsberg 2007); one with 40\% loose heads, one with $18 \%$ pathological heads and one with $20 \%$ mid-piece defects. One mink was sterile as it had sperm which all lacked tail. The seemingly earlier start of sperm production in adult than in juvenile males indicate that the reproductive system is not fully developed during the first year of life.

Taken together, the present study comprises unique data on free ranging mink, which contributes to the characterization of the mink as a sentinel species for monitoring environmental and reproductive health. When assessing reproductive data from wild mink populations, season must be taken into account, but nutritional status does not seem to have a significant influence. Age is also crucial, but the single distinction between juveniles and adults is probably sufficient in order to describe the effect of age.

Acknowledgements The authors would like to thank Karin SellinWretling and Annika Rikberg for their skilled technical assistance. We also thank the Swedish National Veterinary Institute for allowing us in their facilities. This study was financially supported by the Environmental Monitoring Programme at the Swedish University of Agricultural Sciences.

Open Access This article is distributed under the terms of the Creative Commons Attribution Noncommercial License which permits any noncommercial use, distribution, and reproduction in any medium, provided the original author(s) and source are credited.

\section{References}

Aulerich RJ, Sloan CS, Ringer RK (1972) Electroejaculation of mink (Mustela vison). J Anim Sci 34(2):230-233 
Axnér E, Linde Forsberg C (2007) Sperm morphology in the domestic cat, and its relation with fertility: a retrospective study. Reprod Domest Anim 42(3):282-291. doi:10.1111/j.1439-0531.2007.00780.x

Bækgaard H, Larsen PF, Clausen T, Sønderup M (2008) Indflydelse af minktævers huld og huldudvikling på kuldstørrelse (influence of female body-condition on littersize in mink). Annual report, 2008. Danish Fur Breeders Research Center, Holstebro

Basu N, Scheuhammer AM, Bursian SJ, Elliott J, Rouvinen-Watt K, Chan HM (2007) Mink as a sentinel species in environmental health. Environ Res 103(1):130-144. doi:10.1016/j.envres.2006.04.005

Beeby A (2001) What do sentinels stand for? Environ Pollut 112 (2):285-298. doi:10.1016/S0269-7491(00)00038-5

Ben-David M (1997) Timing of reproduction in wild mink: the influence of spawning pacific salmon. Can J Zool/Rev Can Zool 75(3):376-382. doi:10.1139/z97-047

Ben-David M, Flynn RW, Schell DM (1997) Annual and seasonal changes in diets of martens: evidence from stable isotope analysis. Oecologia 111(2):280-291. doi:10.1007/s004420050236

Bergmann C (1847) Über die Verhältnisse der Wärmeökonomie der Thiere zu ihrer Grösse. Göttinger Studien 3(1):595-708

Blottner S, Schon J, Jewgenow K (2006) Seasonally activated spermatogenesis is correlated with increased testicular production of testosterone and epidermal growth factor in mink (Mustela vison). Theriogenology 66(6-7):1593-1598. doi:10.1016/j.theriogenology.2006.01.041

Boissin-Agasse L, Jacquet JM, Lacroix A, Boissin J (1986) Circadian participation in the photoregulation of testis activity and prolactin secretion in the mink, a short-day breeder. J Biol Rhythms 1 (3):231-241. doi:10.1177/074873048600100305

Buskirk SW, Harlow HJ (1989) Body-fat dynamics of the American marten (Martes americana) in winter. J Mammal 70(1):191-193

Calabrese EJ, Aulerich RJ, Padgett GA (1992) Mink as a predictive model in toxicology. Drug Metab Rev 24(4):559-578. doi: $10.3109 / 03602539208996305$

Clark BR, Price EO (1981) Sexual maturation and fecundity of wild and domestic Norway rats (Rattus norvegicus). J Reprod Fertil 63 (1):215-220. doi:10.1530/jrf.0.0630215

Dauphine TC Jr (1975) Kidney weight fluctuations affecting the kidney fat index in Caribou. J Wildl Manage 39(2):379-386

Dunstone N (ed) (1993) The mink. T. \& A. D. Poyser, London

Elder WH (1951) The baculum as an age criterion in mink. J Mammal 32(1):43-50

Enders RK (1952) Reproduction in the mink (Mustela vison). Proc Am Philos Soc 96(6):691-755

Gerell R (1967) Food selection in relation to habitat in mink (Mustela vison Schreber) in Sweden. Oikos 18(2):233-246

Gerell R (1970) Home ranges and movements of the mink (Mustela vison Schreber) in southern Sweden. Oikos 21(2):160-173

Korhonen H, Harri M, Mononen J (1989) Regulation of weight loss in male farm mink. Comp Biochem Physiol A Comp Physiol 92 (3):355-357. doi:10.1016/0300-9629(89)90575-6

Lagerlöf N (1934) Morphological studies on the change in sperm structure and in the testes of bulls with decreased or abolished fertility. Acta Pathol Microbiol Scand 19:254-267
Lechleitner RR (1954) Age criteria in mink, Mustela vison. J Mammal 35(4):496-503

Martinet L, Allain D, Chabi Y (1985) Pineal denervation by cervical sympathetic ganglionectomy suppresses the role of photoperiod on pregnancy or pseudopregnancy, body weight and moulting periods in the mink (Mustela vison). J Endocrinol 107(1):31-39. doi:10.1677/joe. 0.1070031

Martinet L, Mondain-Monval M, Monnerie R (1992) Endogenous circannual rhythms and photorefractoriness of testis activity, moult and prolactin concentrations in mink (Mustela vison). J Reprod Fertil 95(2):325-338. doi:10.1530/jrf.0.0950325

Melero Y, Palazón S, Revilla E, Martelo J, Gosàlbez J (2008) Space use and habitat preferences of the invasive American mink (Mustela vison) in a mediterranean area. Eur J Wildl Res 54 (4):609-617. doi:10.1007/s10344-008-0186-7

Mustonen A-M, Pyykönen T, Paakkonen T, Ryökkynen A, Asikainen J, Aho J, Mononen J, Nieminen P (2005) Adaptations to fasting in the American mink (Mustela vison): carbohydrate and lipid metabolism. Comp Biochem Physiol A Mol Integr Physiol 140 (2):195-202. doi:10.1016/j.cbpb.2004.12.004

Nieminen P, Käkelä R, Mustonen A-M, Hyvärinen H, Asikainen J (2001) Exogenous melatonin affects lipids and enzyme activities in mink (Mustela vison) liver. Comp Biochem Physiol C Toxicol Pharmacol 128(2):203-211. doi:10.1016/S1532-0456(00)00190-3

O'Brien DJ, Kaneene JB, Poppenga RH (1993) The use of mammals as sentinels for human exposure to toxic contaminants in the environment. Environ Health Perspect 99:351-368

Onstad O (1967) Studies on postnatal testicular changes, semen quality, and anomalies of reproductive organs in the mink. Acta Endocrinol Copenh 55(3 Suppl):S9. doi:10.1530/acta.0.055S009

Pilbeam TE, Concannon PW, Travis HF (1979) The annual reproductive cycle of mink (Mustela vison). J Anim Sci 48(3):578-584

Ramos AS, Basrur PK (1972) Seasonal polymorphism of the adrenal cortex in the male mink. Can J Comp Med 36(3):249 255

Sundqvist C, Ellis LC, Bartke A (1988) Reproductive endocrinology of the mink (Mustela vison). Endocr Rev 9(2):247-266. doi:10.1210/edrv-9-2-247

Sundqvist C, Amador AG, Bartke A (1989) Reproduction and fertility in the mink (Mustela vison). J Reprod Fertil 85(2):413-441. doi:10.1530/jrf.0.0850413

Tauson AH (1985) Different feeding intensity levels to mink, 1: effects on male reproductive performance. Swed J Agric Res 15 (2):77-85

Tauson AH (1994) Effect of body condition and dietary energy supply on reproductive processes in the female mink (Mustela vison). In: Concannon PW, England GCW, Verstegen JP, Russell HA (eds) Journal of reproduction and fertility; fertility and infertility in dogs, cats and other carnivores. J Reprod Fertil Suppl 47:37-45

Vuren DV, Coblentz BE (1985) Kidney weight variation and the kidney fat index: an evaluation. J Wildl Manage 49(1):177179 